\title{
ERRATA 2
}

No artigo: Symbiotic and endophytic fungi as biocontrols against cocoa (Theobroma cacao L.) phytopathogens, com número de DOI: $<10.1590 / 0100-5405 / 2175>$, publicado no periódico Summa Phytopathologica, 43(2): 87-93, na 87:

\section{Onde se lia:}

${ }^{3}$ Pontificia Universidad Javeriana Cali, Facultad de Ingeniería, Departamento de Ingeniería Civil e Industrial, Cali, Colombia. Tel.: +57(2) 3218200 ext. 8016. jwescobar@javerianacali.edu.co

\section{Leia-se:}

${ }^{3}$ Universidad del Valle, Facultad Ciencias de la Administración, Departamento de Contabilidad y Finanzas. Cali, Colombia. Tel. +572 5585937. john.wilmer.escobar@correounivalle.edu.co.

Na página 87, em RESUMO onde se lia:

Lima, L.L.; Scaloppi, E.A.G.; Barreto, L.F.; Barreto, M.

Leia-se:

Villamizar-Gallardo, R.A.; Ortíz-Rodriguez, O.O.; Escobar, J.W. 